\title{
ESCENARIOS Y NORMAS DE APLICABILIDAD EN UN MODELO DE ENSEÑANZA Y APRENDIZAJE 2.0 CON EL USO DE BLOGS Y MICROBLOGS
}

\author{
Fernando Checa García \\ Euro-Mediterranean University Institute \\ Universidad Complutense de Madrid
}

http://dx.doi.org/10.5209/rev_NOMA.2014.v44.n4.49296

\begin{abstract}
Resumen.- La utilización de blogs como herramienta para la creación y difusión del conocimiento se ha manifestado en los últimos años como una de las vías más interesantes para el trabajo en entornos de aprendizaje. La capacidad que los blogs ofrecen para el trabajo en las escuelas y las universidades abre vías no solo para una forma de enseñanza y aprendizaje diferente sino para un cambio muy amplio en la relación de los centros educativos con su entorno. En este artículo analizamos los diferentes escenarios de uso que pueden tener los blogs al aplicarse desde un punto de vista metodológico usando un modelo definido, en diferentes entornos educativos. También analizamos y exponemos las diferentes normas de aplicabilidad que un modelo de estas características debe tener en cuenta para la obtención de resultados positivos y mensurables.
\end{abstract}

Palabras Clave.- Blogs, microblogs, enseñanza, aprendizaje, educación, tecnología educativa, centros educativos

Abstract.- The use of blogs as a tool for the creation and dissemination of knowledge has emerged in recent years as one of the most interesting ways to work in learning environments. Working with blogs offer for schools and universities not only open lines for a different way of teaching and learning, but for a large change in the relationship of schools with their environment. In this paper we analyze the different usage scenarios that may have blogs when it applied from a methodological point of view using a model defined in different educational ways. We also analyze and expose different rules than a model with these characteristics should be considered for obtaining positive and measurable results.

Keywords.- Blogs, microblogs, teaching, learning, education, education technology, education centres

\section{Escenarios de uso del modelo Enseñanza-Aprendizaje 2.0}

El modelo que tomamos como base trata de aproximarse al uso de los blogs como herramienta docente (Checa, 2013) y ha sido desarrollado para que pueda incorporarse de forma flexible en cualquiera de los cuatro niveles responsables de la docencia universitaria, el profesorado a título individual, las 
áreas o departamentos, las escuelas y facultades o las universidades en su globalidad, como se puede observar en la Figura 1.

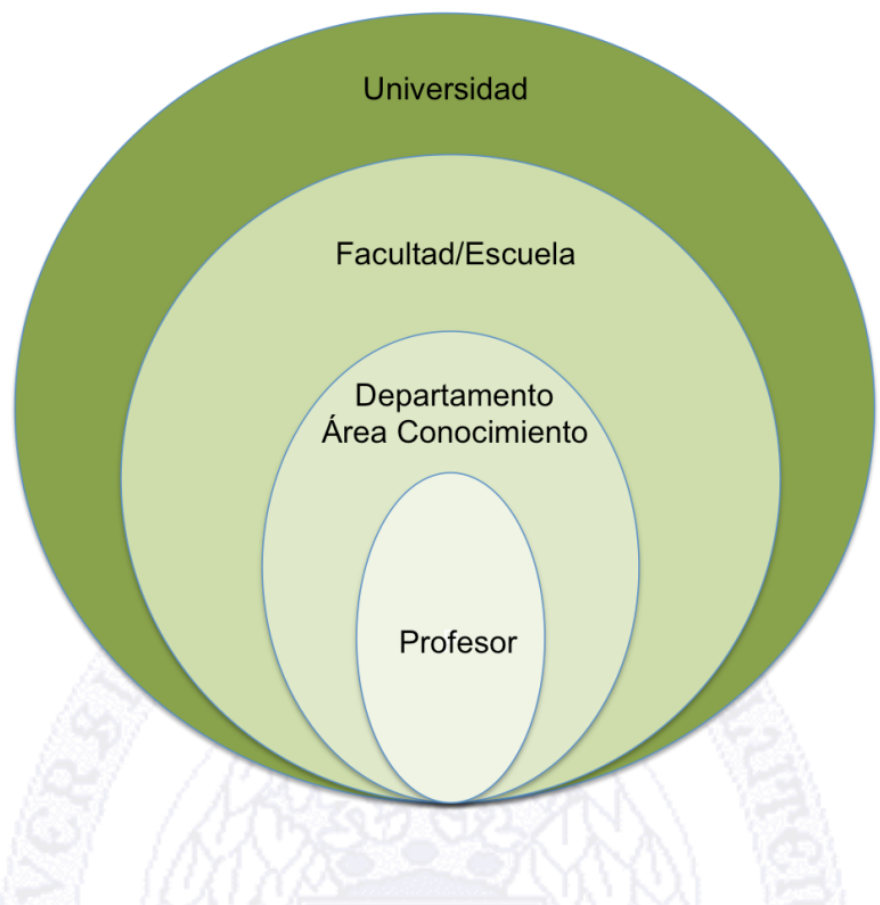

Figura 1. Escenarios de Uso. Enseñanza-Aprendizaje 2.0

Fuente: Elaboración propia

Hay que tomar en consideración que el entorno cambiará de forma sustancial en función de que el modelo sea afrontado desde el trabajo de una asignatura específica o de forma genérica por la universidad, requiriendo un incremento de la coordinación así como de un cambio de paradigma formativo según se vaya generalizando.

\section{Enseñanza-Aprendizaje $\mathbf{2 . 0}$ desde el punto de vista del profesor individual}

Puesto en marcha por un profesor y orientado a la mejora de la docencia de una asignatura o materia concreta, desarrolla sus puntos fuertes en la Fase Introductoria y en la Fase Conversacional, debido a que los profesores que decidan trabajar de forma experimental con el Modelo es muy posible que tengan un conocimiento alto, realicen un uso frecuente y posean elevadas capacidades formativas sobre el uso de las herramientas de blogging y los sistemas de microblogging, así como una elevada orientación en la motivación de la creación de espacios de diálogo y debate entre sus alumnos. Las mayores dificultades pueden producirse en la Fase Evaluativa, tanto por la posibilidad de ser poco comprendida o incluso no aceptada por el resto de la organización, como por la complejidad en la resolución de conflictos en el caso de que se produzcan y no se tenga el apoyo institucional.

En este escenario la carga de trabajo y el protagonismo de la innovación se va a reflejar en el profesor que debe afrontar sin ayudas toda la implantación del 
modelo, y con un riesgo elevado de no obtenerse los resultados deseados en el corto plazo con el consiguiente abandono o desmotivación.

En el escenario individual pueden situarse las pruebas piloto comenzadas de forma personal por profesores con un alto grado de implicación en la aplicación de nuevas técnicas docentes, y la medida del éxito ha de hacerse siempre atendiendo a las limitaciones que pueden aparecer cuando los alumnos consideren que es tan solo una metodología específica de la asignatura, con tiempos acotados y fecha definitiva de finalización.

El uso de blogs en la clase impulsados por profesores de manera puntual está siendo incorporado de forma dispersa en las universidades y la metodología de trabajo es tan variada como los intereses de cada profesor, generándose variabilidades sustanciales entre los distintos procesos según las personalidades, criterios, objetivos y entornos docentes.

\section{Enseñanza-Aprendizaje 2.0 en los departamentos universitarios y áreas de conocimiento}

La puesta en funcionamiento del Modelo de forma coordinada en un departamento $o$ un área de conocimiento determinada permite que la sistematización de los procesos sea aceptada de manera más generalizada. El incremento del número de profesores y estudiantes participantes en el nuevo entorno de trabajo sitúa al Modelo en su entorno más desarrollado al permitir una reflexión conjunta y colaborativa, en parte del claustro, de las estrategias de implantación y ofrece todavía una elevada flexibilidad en las acciones a llevar a cabo.

En este escenario cobra especial importancia la fase introductoria, tanto en lo que respecta a la formación de los profesores con menores habilidades en el uso de las herramientas, o más reacios a su beneficio tangible, como en la generación de estrategias comunes para el diseño de los objetivos pedagógicos y las competencias a desarrollar en cada una de las materias del departamento o el área. La fase evaluativa puede llevarse a cabo sin que se produzca la sensación de cierta soledad del profesor ante los conflictos que puedan sucederse, si bien la conflictividad puede reflejarse en el ámbito departamental, en el caso de que exista una parte del profesorado contraria a la introducción de mecanismos de evaluación pactada.

Las fases reflexiva y conversacional se ven impulsadas en este escenario al aumentar la base de participantes y el Modelo en su conjunto puede desarrollarse de manera óptima al cabo de poco tiempo.

\section{Enseñanza-Aprendizaje 2.0 en las facultades y escuelas universitarias.}

Los ámbitos superiores de puesta en funcionamiento del Modelo se sitúan en su implantación completa en los centros universitarios. En el nuevo entorno que marcan las directrices de aplicación del Espacio Europeo de Educación Superior los centros de las universidades están obligados a afrontar nuevos 
estilos pedagógicos y comunicativos, trasladando de forma activa y continuada sus avances a la sociedad. El Modelo pasa en este caso a revestir mayor complejidad, dado que los recursos utilizados, tanto desde el punto de vista tecnológico, como desde el de las estrategias de cada facultad o escuela. El uso de los blogs como metodología de cambio y la conversación que se genera en ellos debe enfocar los aspectos más genéricos que afectan a cada centro educativo universitario.

En el modelo que proponemos, el centro se convierte en emisor de un nuevo mensaje y en el responsable de su propio espacio de discusión. El blog del centro se plantea en este caso como la solución idónea para alcanzar la nueva identidad on line y mayor visibilidad en Internet de un conglomerado de intereses y participantes tan complejo como variado.

Las fases del modelo son similares a las de los dos escenarios anteriores, volviendo a tener una gran importancia la primera de ellas, formativa y divulgadora de las características del sistema. El desarrollo de los blogs en este escenario ha de contar a su vez con la homogeneización de las herramientas tecnológicas utilizadas. Una vez que el centro ha decidido poner en funcionamiento el Modelo, se debe afrontar la decisión sobre el entorno que se utilizará, atendiendo a los criterios de sencillez, versatilidad, seguridad y coste, sin perder de vista las exigencias de libre acceso y publicación.

También en este escenario la conversación puede expandirse generando situaciones que inicialmente escapen a los controles habituales. La flexibilización de los puntos de control ha de llevarse adelante teniendo presente las especiales características del nuevo entorno.

La fase evaluativa corresponde en este caso, no a los blogs o conversaciones de los alumnos, sino al propio trabajo en el blog y el sistema de microblog, a los contenidos publicados y la frecuencia de publicación, a las interacciones producidas, a los comentarios recibidos o a los enlaces compartidos.

Dada la dificultad de coordinación de un espacio de estas características, el Modelo propone el funcionamiento de pequeños grupos de control formados por representantes de toda la comunidad del centro, profesores, estudiantes, y personal de servicios, que actúen como impulsores del sistema desde un punto de vista centrado en los intereses globales.

\section{Enseñanza-Aprendizaje 2.0 en las universidades}

El marco en el que se integran profesores, departamentos, escuelas y facultades es la universidad, componente fundamental de la formación superior, motor de cambios y de transferencia de conocimiento. Organización que asume sus compromisos con el entorno en el que se integra y situada en un proceso de evolución permanente, entre cuyos últimos aspectos de cambio y adaptación a las nuevas circunstancias se encuentra el Espacio Europeo de Educación Superior. 
Las universidades, con características muy diferentes según su tamaño, ubicación, perfil de sus titulaciones o titularidad pública o privada, no deben ser ajenas a un modelo en el que los profesores y los estudiantes generan nuevas formas de comportamiento y de aprendizaje. Son las universidades desde la estructura institucional las que deben apoyar los mecanismos de cambio producidos desde los primeros eslabones de la cadena educativa, los profesores, hasta sus centros.

Al igual que en el escenario de las escuelas y facultades, la función del Modelo en la institución debe ser la de mostrar la organización de una forma distinta a la reflejada hasta ahora. No sólo servir como escaparate sino como elemento que introduzca y alimente el diálogo, la expresión participativa, el desarrollo de la crítica, desde y hacia la universidad, y la responsabilidad en todos los niveles de relación. Creando y ofreciendo contenidos de valor a su entorno y participando en la conversación global a través del blog y el microblogging "corporativo".

La gran magnitud de las universidades lleva a plantearse dos formas de actuación. En la primera de ellas, el blog tendría una autoría genérica y colaborativa, apareciendo los participantes o editores de cada una de las entradas identificados con su nombre o perfil dentro de la universidad. Este tipo de blog, no obstante, puede correr el riesgo de terminar siendo utilizado por un grupo muy reducido de profesores que actúen como portavoces de la universidad sin que tengan muy claro cuál es su papel dentro del modelo. Por otro lado, la universidad puede incorporar a su blog la voz del rector y el equipo rectoral, como máximos representantes de la organización y transmisores del espíritu de la entidad universitaria.

Al igual que puede ocurrir en el caso de los blogs puestos en funcionamiento en las empresas, en los blogs de las universidades es posible que se genere cierto temor ante la apertura de la conversación libremente y la aparición de situaciones conflictivas en la forma de comentarios excesivamente críticos o que superen la dimensión crítica para realizar ataques directos contra la institución. Por ello deberá crearse un conjunto de protocolos que permitan la conversación minimizando los riesgos ante ataques pero sin renunciar a aceptar la crítica en el caso de que ésta se produzca mostrando claramente cuál es el criterio de aceptación de comentarios elegido.

En este escenario, al contrario que en los del profesorado de forma individual y en los de los departamentos, el proyecto debe afrontarse siempre con un recorrido a largo plazo, enmarcado a su vez en el resto de acciones que permitan la presencia de la universidad en todos los entornos de la web social.

Tras analizar los cuatro escenarios de aplicación del Modelo EnseñanzaAprendizaje 2.0 observamos que el punto óptimo de implementación se encuentra en los entornos departamentales y de área. No obstante, la puesta en funcionamiento por profesores de forma aislada permite tener consciencia de las ventajas del modelo así como de las dificultades ante las que el mismo puede enfrentarse, es interesante para la realización de pruebas piloto y abre el camino para la implantación de nuevos desarrollos en la docencia universitaria. El problema que aparece cuando es el profesor aislado el que incorpora el modelo es que se corre el riesgo citado de que el alumnado considere el 
escenario de trabajo como algo exclusivo de una materia o asignatura, perdiendo valor al diluirse entre todos los cursos y sus requerimientos específicos.

Sin embargo el departamento universitario, conjunto de materias relacionadas entre sí a través de áreas de conocimiento afines, ofrece un paisaje idóneo para la puesta en marcha del modelo. El número de profesores involucrados es mayor y el número de alumnos permite tener una masa crítica como para poder observar el funcionamiento de la acción con vistas a la exportación a otros departamentos que prefieran ser conservadores en los tiempos de implantación. Entendemos que el modelo Enseñanza-Aprendizaje 2.0 se ve forzado por las urgencias que los cambios exigen a las organizaciones, pero también entendemos que el éxito y la minimización de errores parten de la realización de pruebas medibles y valorables.

Los escenarios de centro y organización muestran, como hemos señalado, un cambio de las rutinas organizativas y de las estrategias de comunicación y relación en un plano que supera al trabajo diario con los estudiantes, pero no son por ello menos importantes. La voz de cada centro y de cada universidad, personalizada en una herramienta sencilla y cercana como un blog o un microblog es amplificada a través de los nuevos usos de Internet. La presencia del equipo de gestores como una personalidad cercana y tangible ofrece al entorno, a estudiantes, profesores, padres y en general, todos los sujetos que puedan interactuar con alguna parte de la universidad, un nuevo mensaje de participación. Se rompe la barrera del diálogo a través de los entornos que denominamos 1.0, como los sitios web tradicionales, se abre el espacio a la conversación y se avanza en la transición al uso activo y generalizado de las nuevas herramientas web, desde una óptica de conocimiento libre y abierto a todos.

\section{Las normas de aplicabilidad del modelo}

Los blogs en el entorno educativo pueden aparecer de muchas y variadas formas aunque la puesta en marcha del modelo Enseñanza-Aprendizaje 2.0 no quiere costreñir ni limitar los usos y aplicaciones más comunes, sino más bien incorporarlos y sistematizarlos, buscando generar criterios y normas que puedan generar un marco sistemático para el trabajo con ellos. Todos los modelos ofrecen cierto grado de rigidez y no es nuestra intención generar normativas que creen barreras o dificultades para su uso, sino más bien definir las normas básicas entre las cuales se mueven las experiencias realizadas dentro de la blogosfera educativa y las que consideramos fundamentales de aplicación en nuestro modelo.

Para la generación de un mapa normativo hemos tomado como punto de partida el mapa conceptual de Felipe Zayas reelaborándolo y convirtiéndolo en un mapa que muestre los diferentes escenarios del modelo, los actores principales en el proceso de escritura y edición de los blogs y el enfoque que en cada uno de ellos debería afrontarse. En nuestro mapa de aplicabilidad normativa no existen relaciones cerradas entre los distintos actores y sus características ya que la conversación que se establece en la blogosfera 
gracias al uso de comentarios y enlaces fluye sin límite y sin barreras pudiéndose saltar de uno a otro a través del uso de los hiperenlaces. El mapa normativo aporta al modelo la visibilidad esquematizada de los posibles usos de los blogs y los microblogs siendo de aplicación parcial o totalmente y para su elaboración y mejor comprensión hemos partido de una exposición piramidal, si bien la filosofía de todo el modelo no ha de olvidar el criterio de horizontalización de todos los flujos comunicativos, tal como están siendo desarrollados en el ámbito de la Web 2.0.

El conocimiento, la exposición y transferencia del mismo, es la norma base que tomamos en cuenta en todo el mapa. Y como pieza fundamental para el mismo, el aprendizaje, desde todos los ángulos y segmentos de la universidad, dialogante, critico y compartido entre los agentes que participan de él. Abierto y riguroso e implicado permanentemente en la mejora y la calidad, así como la innovación y su aplicabilidad en los esquemas de cada rama del saber.

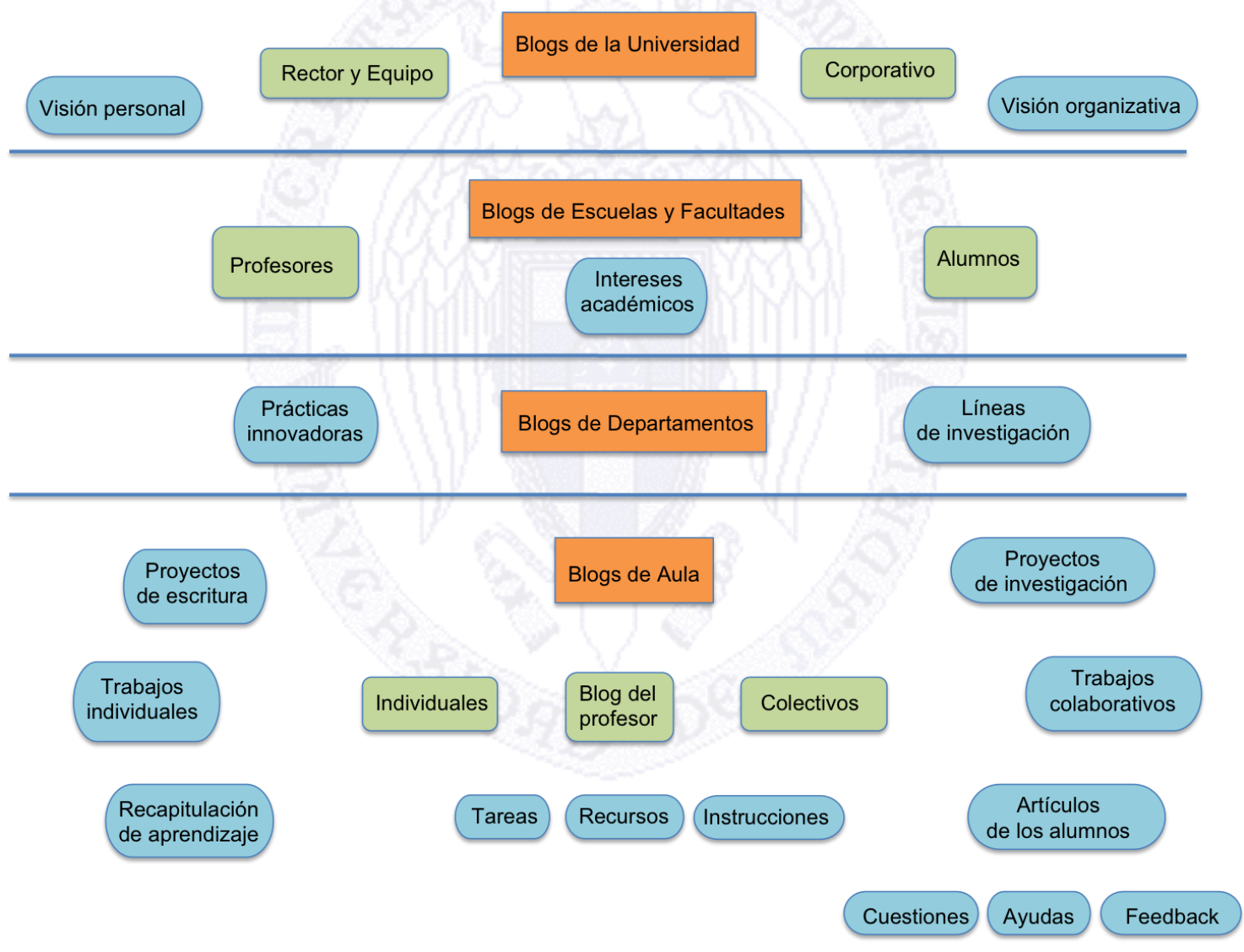

Figura 2. Mapa de aplicabilidad

Fuente: elaboración propia

En el plano superior del mapa situamos a la universidad como organización y la comunicación que se establece desde el ámbito corporativo y desde los responsables y gestores. La universidad adquiere con el blog corporativo una entidad diferenciadora y personal que se ve apoyada con la construcción de los blogs de los responsables como rectores o equipo rectoral. La voz personal y los intereses académicos de los responsables académicos y de gestión se 
suma de esta forma a los desarrollos que empujan la visión de la universidad generando un entorno de cercanía a la sociedad y abriendo la comunicación hacia un plano de flexibilidad y agilidad que no ha de sustituir al trabajo del resto de áreas de gestión como los departamentos de comunicación y marketing, sino más bien acompañar y apoyar su labor comunicativa desde un punto de vista informal y cercano.

Desde las escuelas y facultades el modelo agrupa los blogs de profesores y alumnos con un enfoque centrado en los intereses académicos. Los centros, con su autonomía relativa dentro de la organización universitaria, ofrecen en los blogs asignados una visión enfocada en cada campo de actividad y disciplina. Los avances y el día a día de cada centro se muestra en esos espacios de comunicación de forma sencilla y manejando unos tiempos de publicación así como una agenda de temas diferente a la de las exigencias de la gestión de una gran organización. La libertad de publicación y la agilidad de llevar el mensaje hacia los lectores, así como los nuevos espacios conversacionales creados crean unas escuelas y facultades distintas, ágiles y mucho más enfocadas en el exterior.

Como hemos citado anteriormente, el espacio de máxima expansión del modelo se establece en los departamentos, gracias a la capacidad de coordinación entre los profesores para establecer líneas de actuación respecto a los objetivos de uso, desarrollos y evaluaciones. No obstante los departamentos universitarios pueden implantar con bastante sencillez sus propios blogs colaborativos en los que muestren las prácticas docentes innovadoras, así como las líneas de investigación en las que se está trabajando. Compartir las mejores prácticas con cualquiera que acceda al blog supone ponerlas en un espacio público de discusión que ofrece la posibilidad de mejorarlas o reformarlas si se considera necesario gracias a la colaboración de terceros. De igual manera, hacer partícipes a la comunidad de las líneas de investigación en curso, los aspectos en los que el departamento está trabajando, los grupos de investigadores que están incluidos y sus logros y producción científica es una forma idónea de realizar la transferencia de conocimiento a la sociedad sin dejar de usar las herramientas científicas tradicionales como congresos o publicaciones.

Profesores y alumnos se entrecruzan entre sí en el espacio del aula y su nueva definición. Un aula abierta que desarrolla nuevas prácticas y en las que los blogs de aula, ejemplificados por los del profesor y los de los alumnos, individuales y colectivos, como generadores de conocimiento de forma permanente. Desarrollándose de distinta forma según las diferentes disciplinas y la expresión que cada una de ellas exige. Los blogs de aula, abiertos al libre acceso por parte de cualquier usuario de Internet, ofrecen una adaptación a los géneros, lenguajes, convenciones, reglas y expectativas de cada área de trabajo universitaria. Realizan aportaciones de forma individual a través de los proyectos de escritura y los trabajos de los alumnos, ofreciendo una nueva voz para el aprendizaje y muestran públicamente una recapitulación permanente sobre el aprendizaje conseguido. De igual manera, los blogs de aula también tienen la función de generar un aprendizaje compartido, siendo utilizados para proyectos colaborativos de investigación en los que se expone públicamente 
tanto el desarrollo como las conclusiones, se reciben ayudas on line para la mejora del trabajo y se recibe una retroalimentación permanente.

$Y$ finalmente el blog del profesor, como eje y elemento crucial en todo el modelo, gestionando su espacio como un apéndice de sí mismo, reformando y renovando sus prácticas docentes y estableciendo hacia fuera una nueva identidad académica y profesional, incorpora al modelo la libertad normativa y la creatividad, así como la innovación permanente, manteniendo su protagonismo dentro de las nuevas reglas de autoridad en la comunidad académica, en la que la reflexión y el diálogo escrito se convierten en protagonistas.

\section{Referencias bibliográficas}

BERG, I., ADMIRAAL, W. y PILOT, A., (2006); Designing student peer assessment in higher education: analysis of written and oral peer feedback. Teaching in Higher Education, Volumen 11(2), 135-147.

BLANCO, S., (2005); Los weblogs como herramienta didáctica en el seno de una asignatura curricular, El ecosistema digital: modelos de comunicación, nuevos medios y público en Internet, València, Servei de Publicacions de la Universitat de València, 151-166

CASTAÑEDA, L., (2007); Software Social para la escuela 2-0: más allá de los Bogs y las Wikis, Inclusión Digital en la educación superior: desafíos y oportunidades en la sociedad de la información, $X$ Congreso Internacional EDUTEC, Buenos Aires

CASTAÑO GARRIDO, C. y PALACIO, G., (2006); Edublogs para el aprendizaje contínuo en la Web Semántica, E-Actividades, un referente básico para la formación en Internet, 95-111, Sevilla: Mad,

-, (2008); Prácticas educativas en entornos Web 2.0, Madrid: Síntesis

CEREZO, J.M. (2206); La blogosfera hispana: pioneros de la cultura digital, Madrid: Fundación France Telecom

CHAMORRO, R., (2008); Blogs. Madrid: Creaciones Copyright

CHECA, F., JOYANES, L., (2009); El uso de los blogs como metodología activa de aprendizaje: Innovando en Gestión del Conocimiento en VI Jornadas Internacionales de Innovación Universitaria. Universidad Europea de Madrid

CHECA, F. (2009); The use of blogs as key element in the new TeachingLearning process" en Nomads, Mediterranean Perspectives, $\mathrm{n}^{\circ} \mathbf{2}$, , MadridMéxico: Paza \& Valdés

- (2013); La utilización del microblogging y de twitter como herramienta de enseñanza-aprendizaje en Espiral, Cuadernos del Profesorado, Vol. 6(11)

- (2013), Introducción a un modelo de docencia-aprendizaje basado en el uso de blogs y microblogs. En Sánchez, J.; Ruiz, J. y Sánchez, E. (Coords.). 
Buenas prácticas con TIC en la investigación y la docencia. Málaga: Universidad de Málaga.

COSTA, C., BEHAM, G. y REHINHARDT, W. (2008); Microblogging In Technology Enhanced Learning: A Use-Case Inspection of PPE Summer School 2008 en Workshop at the European Conference on Technology Enhanced Learning (ECTEL), Maastricht

GARCÍA AGUILERA, F.J. y AGUILAR CUENCA, D. (2002); Ingeniería de la formación: cómo diseñar acciones de formación presenciales en base a criterios de calidad pedagógica, Capital humano: revista para la integración y desarrollo de los recursos humanos, Año número 15, Número 152, 40-47

- et Al, (2004). Metodología didáctica aplicada: estrategias de intervención pedagógica, Málaga: Lavante

GONZÁLEZ SERNA, J. L., (2003); Weblog y Enseñanza, Perspectiva CEP. Revista de los centros del profesorado de Andalucía, Número 6, Noviembre

JIMÉNEZ, R. y POLO, F., (2007); La Gran Guía de los Blogs. Barcelona: Elcobre Ediciones

LARA, T., (2009); El papel de la Universidad en la construcción de su identidad digital. En: "Cultura digital y prácticas creativas en educación" [monográfico en línea]. Revista de Universidad y Sociedad del Conocimiento (RUSC). Vol. 6(1) UOC.

MARTí, D., (2008); Blogs educativos de comunicación en lberoamérica, Revista Diálogos de la Comunicación, Número 76, Enero-Junio.

MARZAL, M. A., y BUTERA, M. J., (2007); Los blogs en el nuevo modelo educativo universitario: posibilidades e iniciativas, BiD, Textos universitaris de biblioteconomia i documentació, Facultad de Biblioteconomia y Documentación, Universitad de Barcelona, número 19. 